\title{
Pseudomonas aeruginosa in patients hospitalised for COPD exacerbation: a
} prospective study

\author{
C. Garcia-Vidal*, P. Almagro*, V. Romaní*, M. Rodríguez-Carballeira*, E. Cuchi”, \\ L. Canales ${ }^{\ddagger}$, D. Blasco ${ }^{+}$, J.L. Heredia ${ }^{\S}$ and J. Garau*
}

ABSTRACT: Risk factors for Pseudomonas aeruginosa (PA) isolation in patients hospitalised for chronic obstructive pulmonary disease (COPD) exacerbation remain controversial. The aim of our study was to determine the incidence and risk factors for PA isolation in sputum at hospital admission in a prospective cohort of patients with acute exacerbation of COPD.

We prospectively studied all patients with COPD exacerbation admitted to our hospital between June 2003 and September 2004. Suspected predictors of PA isolation were studied. Spirometry tests and 6-min walking tests were performed 1 month after the patients were discharged. Highresolution computed tomography (HRCT) was performed in a randomised manner in one out of every two patients to quantify the presence and extent of bronchiectasis. Patients were followed up during the following year for hospital re-admissions.

A total of 188 patients were included, of whom 31 (16.5\%) had PA in sputum at initial admission. The BODE (body mass index, airflow obstruction, dyspnoea, exercise capacity) index (OR 2.18, $\mathrm{Cl}$ $95 \%$ 1.26-3.78; $p=0.005$ ), admissions in the previous year (OR 1.65, Cl 95\% 1.13-2.43; $p=0.005$ ), systemic steroid treatment (OR 14.7, $\mathrm{Cl} 95 \%$ 2.28-94.8; $p=0.01$ ), and previous isolation of PA (OR 23.1, $\mathrm{Cl}$ 95\% 5.7-94.3; $\mathrm{p}<0.001$ ) were associated with PA isolation. No relationship was seen between bronchiectasis in HRCT and antibiotic use in the previous 3 months.

PA in sputum at hospital admission is more frequent in patients with poorer scoring on the BODE index, previous hospital admissions, oral corticosteroids and prior isolation of PA.

KEYWORDS: BODE index, bronchiectasis, chronic obstructive pulmonary disease, hospitalisation, Pseudomonas aeruginosa infection

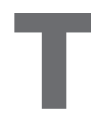
he role of bacterial infection in chronic obstructive pulmonary disease (COPD) exacerbation remains controversial [1,2]. Recent studies have correlated COPD exacerbation with the overgrowth of the bacterial load or with the acquisition of a new strain of pathogenic bacteria [3-5]. In ambulatory patients, Haemophilus influenzae, Moraxella catarrhalis and Streptococcus pneumoniae are the three major pathogens isolated in COPD exacerbations, while Pseudomonas aeruginosa (PA) is uncommon and is usually associated with the greatest degree of functional impairment [6-10]. Hospitalised patients for acute exacerbation of COPD (AECOPD) usually have more advanced disease [11], and the infecting pathogens could be different.
Recent European guidelines highlight the need for specific studies on risk factors for PA in COPD exacerbated patients [12]. This is an important issue because empirical antibiotic regimens designed to cover this pathogen are different from those aimed to cover for the usual microorganisms. Nevertheless, contradictory results regarding the role of PA in sputum at admission in COPD hospitalised patients have been reported [13-15]. Our hypothesis was that there would be specific risk factors that would predict a change in flora and an increased risk for the presence of PA in sputum in this population. On this basis, the aim of the study was to determine the incidence and risk factors for PA isolation in sputum at hospital admission in a prospective cohort of patients with AECOPD.

\section{AFFLLIATIONS}

*Services of Internal Medicine, "Microbiology,

"Radiology,

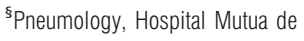
Terrassa, University of Barcelona, and ${ }^{+}$Hospital Clinic, Dept of Clinical Microbiology, Hospital Mutua de Terrassa, University of Barcelona, Barcelona, Spain.

\section{CORRESPONDENCE}

C. Garcia-Vidal

Service of Internal Medicine

Hospital Mutua de Terrassa

Plaza Dr. Robert 5

08221 Terrassa

Barcelona

Spain

E-mail: carolgv75@hotmail.com

Received:

Jan 082009

Accepted after revision:

March 292009

First published online:

April 222009 


\section{METHODS}

\section{Subjects}

We prospectively studied all consecutive patients admitted to our institution (Hospital Mutua de Terrassa, Barcelona, Spain) for AECOPD between June 2003 and September 2004. All episodes of hospital re-admissions of the cohort during the following year, until September 2005, were also prospectively followed up. The study was carried out in a 500-bed university hospital. Inclusion criteria were: hospitalisation for AECOPD, basal forced spirometry showing a forced expiratory volume in $1 \mathrm{~s}$ (FEV1) $\leqslant 70 \%$ of their reference value, and $\beta_{2}$-agonist reversibility of predicted FEV1 of $<15 \%$ and/or $200 \mathrm{~mL}$, with FEV1/forced vital capacity $(\mathrm{FVC})<70 \%$. Exclusion criteria included a history of asthma or bronchiectasis as a predominant illness, pneumonia or pulmonary oedema at admission, hospitalisation for causes other than AECOPD, or patient refusal to participate in the study. COPD exacerbation was defined following the criteria of ANTHONISEN et al. [16]. Admission criteria were at the discretion of the emergency room physician. For the purposes of this study, patients were divided into two groups: those in whom PA was isolated in sputum at hospital admission (PA group) and those in whom PA was not isolated in sputum at hospitalisation (non-PA group). Written informed consent was obtained from each subject and the study was approved by the ethics committee at the Hospital Mutua de Terrassa.

\section{Clinical evaluation}

At the initial visit, patients provided a complete clinical history and underwent a physical examination. Information collected included demographic characteristics, body mass index, comorbidity (as measured by the Charlson index), previous functional dependence (Katz score) and dyspnoea measured by the modified Medical Research Council (mMRC). Information on smoking history, number of hospitalisations for COPD within the last year, time to last hospital discharge, use of antibiotics within the last 3 months and prior admission, use of systemic or inhaled corticosteroids, and chronic home use of oxygen therapy prior to admission were also collected. Chronic systemic corticosteroid use was considered when doses equivalent to prednisone $\geqslant 5 \mathrm{mg} \cdot \mathrm{day}^{-1}$ had been given for at least the previous 3 months.

\section{Microbiological studies}

Spontaneous or induced sputum samples were collected at admission and at each re-admission, before antibiotic administration (section 1 of supplementary data). Bacterial agents were classified into potential pathogenic microorganisms (PPMs) and non-PPMs, as previously described [3]. Only PPMs were evaluated. In some patients with at least two isolates of PA in sputum at different admissions, strains were typed by pulsed-field gel electrophoresis. The presence of a mucoid phenotype on PA isolation agar plates was recorded.

\section{High-resolution computed tomography evaluation}

The presence of bronchiectasis was assessed at the first hospital admission. To limit radiation and costs, patients were randomised for a high-resolution computed tomography (HRCT) of the chest, in a 2:1 ratio. The diagnosis of bronchiectasis was based on standard criteria. HRCT scans were interpreted by two experienced radiologists blinded to the patients' clinical grouping and microbiological status. Posterior consensus was reached in case of disagreement. The bronchiectasis score is detailed in section 2 of the supplementary data.

\section{Follow-up}

A follow-up visit took place $\sim 1$ month after discharge. At this visit, forced spirometry and bronchodilatador testing were performed according to standard techniques [17]. The 6-min walking test was performed following the American Thoracic Society recommendations [18]. The BODE (body mass index, airflow obstruction, dyspnoea, exercise capacity) index was also calculated as the sum score proposed by CELLI et al. [19]. In case of hospital re-admission within the first month after discharge, the patient was followed up for 1 month after reaching clinical stability. All patients were followed up for hospital re-admission during the year after discharge.

\section{Statistical analysis}

Sample size calculation was based on FEV1 values; postbronchodilator FEV1 $40 \%$ of predicted for PA patients, and $50 \%$ for non-PA patients, with an $\alpha$ - and $\beta$-error of 0.05 and 0.1 , respectively. A $10 \%$ patient loss was assumed. Accordingly, the calculated size was 234 patients. To assess factors associated with PA isolation, we compared the PA and nonPA groups. To detect significant differences between groups we used the Chi-squared test with continuity correction for categorical variables. Quantitative variables were analysed using an unpaired t-test or their corresponding nonparametrical tests when the distribution of data so required. The relationship between bronchiectasis score and FEV1 was calculated with the Pearson correlation coefficient. For multivariate analysis a logistic regression model was constructed with PA isolation as a dependent variable. In this model independent variables included the most clinically relevant variables that were found to be significant in bivariate analysis.

Data analysis was performed using the SPSS for Windows software package (version 11; SPSS Inc., Chicago, IL, USA). In all analyses, we considered p-values $\leqslant 0.05$ to be statistically significant. All reported p-values are two-tailed.

\section{RESULTS}

\section{Patient characteristics}

Over the study period, a total of 254 patients with a suspected diagnosis of AECOPD were admitted to hospital. Of these, 66 patients were excluded for the following reasons: impossibility to perform spirometry or lack of spirometric criteria (26 (10.2\%) patients); pneumonia (25 $(9.8 \%)$ patients); bronchiectasis as a main manifestation of disease (six (2.3\%) patients); idiopathic fibrosis (four (1.6\%) patients); and others (five $(2.0 \%)$ patients). The studied population were predominantly male $(95 \%)$, with a mean \pm SD age of $72.1 \pm 10.0$ yrs and length of stay of $11 \pm 8.7$ days. Sociodemographic and functional characteristics are shown in table 1 .

Of the 188 patients included, 157 (83.5\%) were in the non-PA group and $31(16.5 \%)$ were in the PA-group. When comparing groups, no significant differences in age or sex were observed. PA isolation in sputum was more frequent in patients with the worst values of Katz score (4.7 versus $5.6 ; \mathrm{p}=0.03$ ) and the mMRC (3.4 versus $2.7 ; \mathrm{p}=0.001)$. The PA group had a stronger 


\begin{tabular}{|c|c|c|}
\hline TABLE 1 & \multicolumn{2}{|c|}{$\begin{array}{l}\text { Characteristics of patients hospitalised with } \\
\text { acute chronic obstructive pulmonary disease } \\
\text { exacerbation during the period of study }\end{array}$} \\
\hline Subjects $n$ & & 188 \\
\hline Age yrs & & $72.11 \pm 10.0$ \\
\hline Male sex & & $178(94.7)$ \\
\hline PBD FEV 1 L & & $1.04 \pm 0.37$ \\
\hline PBD FEV $1 \%$ & pred & $44 \pm 14.52$ \\
\hline \multicolumn{3}{|c|}{ Severity according to GOLD stage } \\
\hline Stage II, m & derate & $65(34.6)$ \\
\hline Stage III, s & vere & $95(50.5)$ \\
\hline Stage IV, $\mathrm{V}$ & y severe & $28(14.9)$ \\
\hline Walking test & & $330 \pm 105$ \\
\hline Charlson inc & & $2.17 \pm 1.3$ \\
\hline Katz score & & $5.46 \pm 1.3$ \\
\hline Dyspnoea $\mathrm{m}$ & $\mathrm{ARC}$ & $2.78 \pm 1.2$ \\
\hline Smoking pa & r-yrs & $59.84 \pm 35.4$ \\
\hline Patients witl & a hospital admission in the previous month & $32(17)$ \\
\hline Episodes of & dmission in previous year & $1.27 \pm 2.0$ \\
\hline Days hospit & ised in previous year & $12.84 \pm 25.9$ \\
\hline Antimicrobia & $s$ in last 3 months & $62(33)$ \\
\hline Body mass & dex & $27.8 \pm 5.2$ \\
\hline Inhaled ster & & $140(76.5)$ \\
\hline Systemic st & oids & $17(9.3)$ \\
\hline
\end{tabular}

Data are presented mean \pm SD or $n(\%)$, unless otherwise specified. PBD: postbronchodilator; FEV 1 : forced expiratory volume in $1 \mathrm{~s}$; \% pred: \% predicted; GOLD: Global Initiative for Chronic Obstructive Pulmonary Disease; mMRC: modified Medical Research Council.

history of smoking than patients in the non-PA group (mean pack-years of smoking 73.7 versus 56.8; $\mathrm{p}=0.02$ ). Previous hospital admissions in the last month (32.3\% versus $14.0 \%$; $\mathrm{p}=0.001$ ) and number of previous episodes of admission within the last year ( 3.1 versus $0.9 ; \mathrm{p}=0.002$ ) were more frequent in the PA group. Acute and chronic steroid therapy were more frequent in patients in the PA group (22.6\% versus $6.6 \%(p=0.012)$ and $12.9 \%$ versus $2.5 \%(p<0.01)$, respectively). No relationship was found between PA isolation and the use of inhaled steroids or antimicrobial use in the last 3 months.

Respiratory parameters significantly associated with the presence of PA in sputum at admission were: severity of disease as measured by post-bronchodilator FEV1 (mean of FEV1 38.7\% versus $45.9 \%$; $p=0.012$ ), the poorest values for the 6-min walking test $(217.5 \mathrm{~m}$ versus $343.7 \mathrm{~m} ; \mathrm{p}<0.001)$ and chronic home oxygen therapy (32.3 versus 15.6; $\mathrm{p}=0.041$ ). The BODE index was also significantly associated with PA isolation (7.3 versus $5.4 ; p=0.0005)$. As a summary, data of significant variables in bivariate analysis are shown in table 2.

A HRCT scan of the chest was performed in 88 randomised patients. Patients with HRCT were similar with respect to age, previous admissions, corticosteroid use, FEV1, FVC and other measured physiological parameters, compared to those who did not undergo HRCT scanning. 46 (52\%) patients had significant detectable bronchiectasis on HRCT (two or more dilated bronchi; global score in percentage $\geqslant 5.6 \%$ ). Of those in whom bronchiectasis was detected, the median (range) score was 25 (5-56)\% (fig. 1). No statistical relationship was seen between the total bronchiectasis score and FEV1 measurement $\left(r^{2}=0.059 ; \mathrm{p}=0.058\right), 6$-min walking test $\left(\mathrm{r}^{2}=0.002 ; \mathrm{p}=0.784\right)$ or BODE index $\left(r^{2}=0.009 ; p=0.94\right)$. Similarly, no relationship was found between the score of bronchiectasis and positive bacterial culture $(p=0.76)$ or PA isolation in sputum at admission (95\% CI 0.99-1.05; $\mathrm{p}=0.09$ ).

\section{Microbiological findings}

Of the 188 patients included, 119 (63.3\%) had good quality sputum in the first hospital admission. Non-PPMs were isolated in 55\% of these patients while PPMs were found in $45 \%$ of cases. A single bacterial species was isolated in 50 patients, two species in three patients, and three species in one patient. PA was the most frequently isolated species in patients with valid sputum (31 (26\%) out of 119 cases) followed by $S$. pneumoniae and $H$. influenzae (11 (9.2\%) cases each). The presence of other microorganisms was infrequent.

During the initial admission and the subsequent year of prospective follow-up, a total of 469 episodes of hospitalisation due to COPD exacerbation were collected (134 in PA group and 335 in non-PA group), and valid sputum was collected in $220(47 \%)$ episodes. Patients with positive bacterial cultures of sputum (any microorganism) had a lower FEV1 than patients with negative sputum cultures at admission $(p=0.003)$.

As shown in figure 2, the global incidence of PA isolation in the index of hospitalisation and re-admissions during the subsequent year was $23.18 \%$ of all episodes. H. influenzae $(11 \%)$ and S. pneumoniae $(10 \%)$ remained common aetiologies for COPD exacerbation in patients requiring hospitalisation. Patients in the PA-group were re-admitted more frequently than patients in the non-PA group $(p=0.001)$, and were more likely to present valid sputum during these re-admissions than patients in the non-PA group $(\mathrm{p}<0.001)$. The relationship between different microorganisms and FEV1 is shown in figure 3 .

Among the 31 patients with PA at the first admission, PA was isolated in 12 patients only once, twice in 11 patients, and in eight patients at least three times, in sputum cultures performed during subsequent admissions. Previous isolation of PA was associated with a higher probability of a new PA isolation $(p<0.001)$. Molecular typing of 41 PA strains from 10 patients obtained in different exacerbations showed that the current strain was identical to the original strain in seven (70\%) patients and in 37 (90\%) of the samples. Of note, all persisting PA strains were non-mucoid (six patients) with a single instance of persisting mucoid strain (one patient) (see supplementary data). Table 3 shows the antibiotic susceptibility pattern of PA isolates. Of the 31 patients in the PA group, only four patients received empirical antibiotic treatment with pseudomonal coverage (quinolones: $n=3$, ceftazidime: $n=1)$. 12 patients received antipseudomonal treatment when the microbiological results were known.

Factors associated with PA isolation in multivariate analysis Table 4 summarises the results of multivariate analysis of factors potentially associated with PA isolation. Significant variables associated independently with $\mathrm{PA}$ isolation were BODE index (OR 2.18, 95\% CI 1.26-3.78; $\mathrm{p}=0.005$ ), number of 
TABLE 2 Characteristics of 188 patients hospitalised with acute chronic obstructive pulmoanry disease ${ }^{\#}$

\begin{tabular}{|c|c|c|c|c|}
\hline & PA group & Non-PA group ${ }^{+}$ & p-value & OR $(95 \% \mathrm{Cl})$ \\
\hline Katz index & $4.74 \pm 1.9$ & $5.61 \pm 1.1$ & 0.03 & $0.67(0.51-0.90)$ \\
\hline Smoking pack-yrs & $73.7 \pm 39.7$ & $56.76 \pm 33.8$ & 0.02 & $1.01(1.003-1.02)$ \\
\hline Hospital admission in last month & $10(32)$ & $22(14)$ & 0.001 & $2.95(1.16-7.48)$ \\
\hline Admissions in previous year & $3.06 \pm 3.5$ & $0.91 \pm 1.3$ & 0.002 & $1.47(1.19-1.81)$ \\
\hline Systemic corticosteroids & 7 (22.6) & $10(6.6)$ & 0.01 & $3.57(1.17-10.88)$ \\
\hline Chronic systemic corticosteroids & $4(12.9)$ & $4(2.5)$ & 0.01 & $5.66(1.3-24)$ \\
\hline PBD FEV $1 \%$ & $38.7 \pm 12.2$ & $45.9 \pm 14.7$ & 0.01 & $0.96(0.93-0.99)$ \\
\hline Walking test $\mathbf{m}$ & $217.5 \pm 103$ & $343.7 \pm 98.5$ & $<0.001$ & $0.99(0.98-0.99)$ \\
\hline BODE index & $7.32 \pm 1.72$ & $5.42 \pm 2.53$ & $<0.001$ & $1.45(1.17-1.78)$ \\
\hline Previous isolation PA & 19 (61.3) & $12(7.6)$ & $<0.001$ & $122(25.5-590)$ \\
\hline Home oxygen therapy & 10 (32.3) & $24(15.3)$ & 0.04 & $0.48(0.19-1.1)$ \\
\hline
\end{tabular}

Data are presented as mean \pm SD or $n(\%)$, unless otherwise stated. PA: Pseudomonas aeruginosa; mMRC: modified Medical Research Council; PBD: postbronchodilator; FEV1: forced expiratory volume in $1 \mathrm{~s}$; BODE: body mass index, airflow obstruction, dyspnoea, exercise capacity. ${ }^{\#}$ : PA group compared with 157 patients in the non-PA group; ${ }^{\bullet}: n=31 ;{ }^{+}: n=157$.

hospital admissions in the previous year (OR 1.65, 95\% CI 1.13-2.43; $\mathrm{p}=0.005)$, systemic steroid treatment (OR 14.7, 95\% CI 2.28-94.8; $\mathrm{p}=0.01$ ), and previous isolation of PA (OR 23.1, 95\% CI 5.7-94.3; p<0.001).

\section{DISCUSSION}

In this prospective study we offer a comprehensive evaluation of the incidence and risk factors for PA isolation in a large

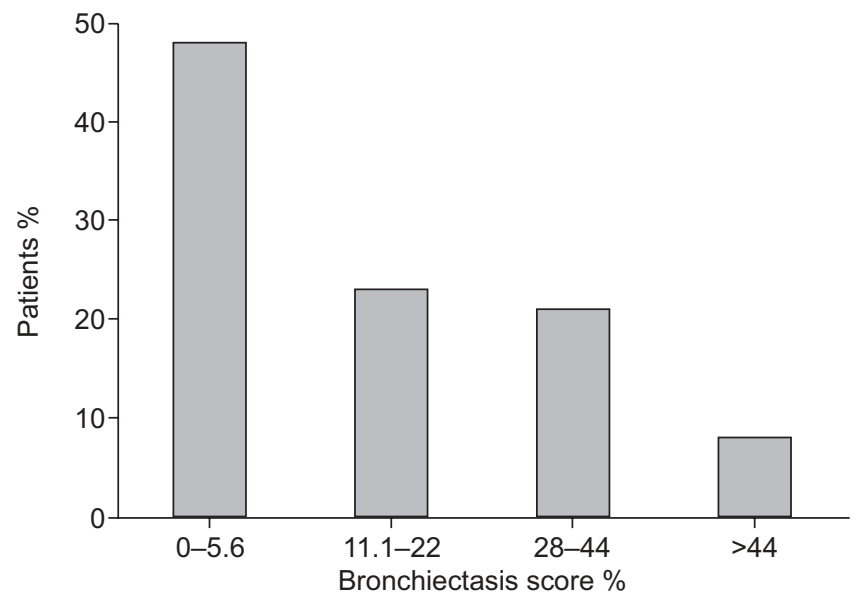

FIGURE 1. Total bronchiectasis score in 88 patients. In total, 46 (52\%) patients had significant detectable bronchiectasis (two or more dilated bronchi; global score in percentage $\geqslant 5.6 \%$ ) on high-resolution computed tomography. The overall bronchiectasis score was expressed as a percentage: (Brochiectasis score / bronchiectasis maximum score) $\times 100$. Patients with a score of 0 or 5.6 (less than two affected segments) were considered normal. prospective cohort of patients hospitalised for AECOPD. To our knowledge, this study is the first to assess specifically, in a prospective cohort, the multidimensional risk factors of PA isolation in sputum in patients hospitalised for AECOPD. The most important finding of our study is the strong relationship between PA isolation at hospital admission and several markers of respiratory functional impairment. Additionally, our study shows that the incidence of PA in sputum in this population is high (23\% of total episodes). Specifically, this

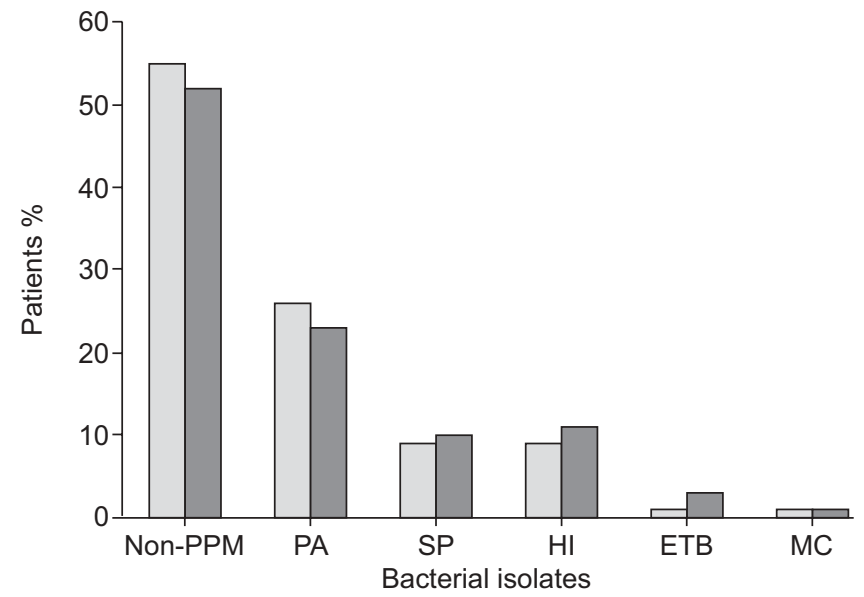

FIGURE 2. Bacterial isolates of patients with valid sputum. al index admission $(\mathrm{n}=119)$; $\mathbb{E}$ : total admissions ( $\mathrm{n}=220$ ). Non-PPM: non-potential pathogenic micro-organisms; PA: Pseudomonas aeruginosa; SP: Streptococcus pneumoniae; HI: Haemophilus influenzae; ETB: enterobacteriaceae; MC: Moraxella catharralis. 


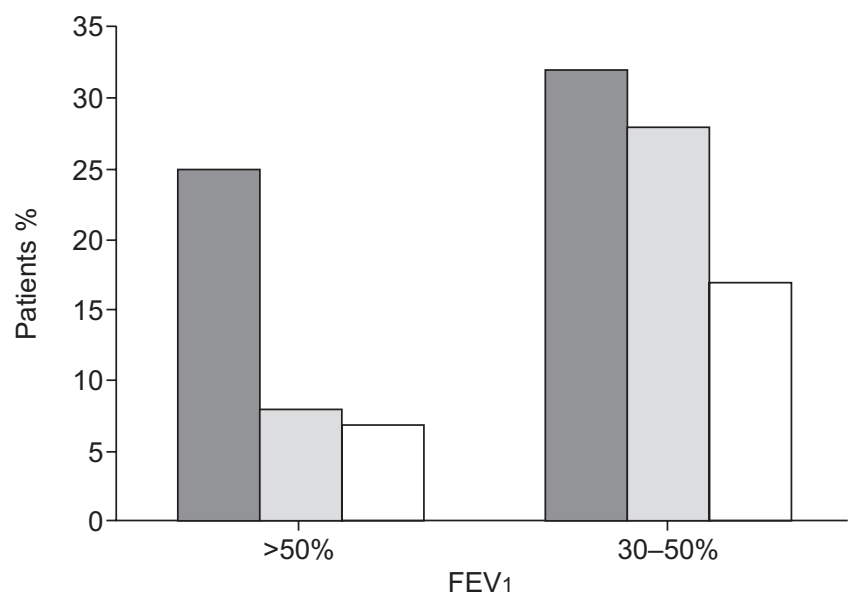

FIGURE 3. The relationship between forced expiratory volume in $1 \mathrm{~s}$ (FEV1) and bacterial isolates in the study patients. non-potential pathogenic microorganisms; $\square$ : Pseudomonas aeruginosa plus enterobacteriaceae; $\square$ : Haemophilus influenzae plus Streptococcus pneumoniae plus Moraxella catharralis. $\mathrm{p}=0.035$.

incidence is higher than that reported in ambulatory patients $[5,8-10]$.

Various studies regarding the microbiology of hospitalised patients for AECOPD have recently been published. ELLER et al. [13] showed the relevance of Gram-negative bacilli isolation in sputum as the most frequent species isolated in a cohort of 112 patients (48.2\% of cultures positive for PPMs). More recently, LIN et al. [20] showed similar results. However, both studies were retrospective and with a likely selection bias (higher rate of sputum collection among patients with more severe disease or no antibiotic response during hospitalisation). In contrast, GROENEWEGEN et al. [14] only reported PA isolation in 15\% of positive cultures (13 out of 85 ) and $18 \%$ of cultures positive for PPMs (13 out of 71). The present study also confirms previous data $[2,8,9,13,15]$ reporting a higher incidence of bacterial isolation in sputum in patients with worse values of FEV1.

The relationship between the type of bacteria isolated and the degree of functional impairment, as measured by FEV1, has been a matter of debate in recent years. ELLER et al. [13] reported a correlation between lower FEV1 values and the presence of PA in patients hospitalised for AECOPD. In contrast, GROENEWEGEN et al. [14] were not able to demonstrate differences between the type of bacteria isolated and clinical characteristics or lung functional parameters. A possible explanation is that GROENEWEGEN et al. [14] performed spirometry tests prior to discharge from hospital and, therefore, they should not be considered true baseline values.

The risk factors for PA isolation in sputum in patients with AECOPD have become a major issue. Although guidelines offer recommendations for the subset of patients in whom PA should be strongly suspected [12, 21], limited data exist to identify this population, and the available data come mostly from studies involving ambulatory patients $[8,10]$. To date, the accepted risk factors for PA isolation in hospitalised patients were previous hospitalisation, recent antibiotic therapy, disease severity measured with FEV1 and previous infection

\begin{tabular}{ll} 
TABLE 3 & $\begin{array}{l}\text { Antibiotic resistance profile of } 31 \text { Pseudomonas } \\
\text { aeruginosa isolates }\end{array}$ \\
Antibiotic & Number of susceptible strains (\%) \\
\hline Ciprofloxacin & $24(77.4)$ \\
Piperacillin-tazobactam & $26(83.9)$ \\
Ceftazidime & $27(87.1)$ \\
Imipenem & $27(87.1)$ \\
Gentamicin & $25(80.6)$ \\
Amikacin & $27(87.1)$ \\
\hline
\end{tabular}

with PA. The present study confirms some of these risk factors, and adds new prognostic variables such as functional dependence, dyspnoea score, walking test, oral corticosteroid treatment and, of note, the BODE index. This multidimensional index is an excellent marker of severity in COPD and provides more reliable information about vital prognosis, risk of hospitalisation and treatment response than FEV1 [19, 22, 23]. Additionally, the BODE index, along with systemic steroids treatment, hospital admissions during the previous year and previous isolation of PA, are independent factors associated with the isolation of PA in sputum in AECOPD.

Our study was not able to demonstrate a relationship between antibiotic treatment in the last 3 months and the presence of PA in sputum. This result is concordant with previous studies [14].

One of the most important points of criticism in studies dealing with AECOPD is the inclusion of patients with bronchiectasis. Bronchiectasis is thought to be a special entity. However, two recent studies based on 44 and 110 outpatients, respectively, yielded evidence that moderate bronchiectasis on HRCT is common in ambulatory COPD patients, although studies dealing with the incidence of bronchiectasis in hospitalised patients with COPD were lacking $[24,25]$. We can confirm that the prevalence of moderate bronchiectasis is high in this population $(52 \%)$. However, no relationship was seen between the bronchiectasis score and spirometric measurements or the presence of PA in the sputum. Although we cannot rule out a shortfall of statistical power, these results are similar to previous studies [24].

One of the limitations of our study was the lack of distinction between bacterial colonisation of the lower airways and infection by PA, and this issue remains unsolved. Recently, MuRPHY et al. [26] showed that isolation of a new strain of PA in ambulatory patients was associated with the occurrence of an exacerbation. In their study most new strains of PA $(54 \%)$ cleared up, and in the majority of cases (67.7\%) without active antibiotic therapy. Colonisation, understood as persistent carriage, was observed in only $23 \%$ of patients [26]. Some studies would suggest that the use of quantitative cultures is useful to distinguish between infection and colonisation, but no conclusive results are available [8, 14]. Many studies have used semiquantitative methods to evaluate bacterial infection in AECOPD and, more importantly, recent work has shown that bacteria obtained from good quality sputum are the same as those obtained through bronchoscopy and protected specimen brush [27, 28]. Another limitation of our study was the significant number of patients in the control group (non-PA 


\begin{tabular}{|c|c|c|c|}
\hline \multirow{2}{*}{$\begin{array}{l}\text { TABLE } 4 \\
\text { Variable }\end{array}$} & \multicolumn{3}{|c|}{$\begin{array}{l}\text { Risk factors associated with Pseudomonas } \\
\text { aeruginosa isolation in sputum in } 188 \text { patients } \\
\text { hospitalised with chronic obstructive pulmonary } \\
\text { disease exacerbation: multivariate analysis }\end{array}$} \\
\hline & & p-value & OR (95\% Cl) \\
\hline \multicolumn{2}{|l|}{ BODE index } & 0.005 & $2.18(1.26-3.78)$ \\
\hline \multicolumn{2}{|c|}{ Hospital admissions in previous year } & 0.005 & $1.65(1.13-2.43)$ \\
\hline \multicolumn{2}{|c|}{ Systemic steroid treatment } & 0.01 & $14.7(2.28-94.8)$ \\
\hline \multicolumn{2}{|c|}{ Previous isolation of $P$. aeruginosa } & $<0.001$ & $23.1(5.7-94.3)$ \\
\hline
\end{tabular}

group) without a valid sample of sputum, and the low rate of isolation of PPMs. Nonetheless, the percentage of positive sputum samples was similar to that found in previous prospective studies $[15,28]$, and the aim of our study was to determine the risk factors for PA isolation in patients hospitalised with AECOPD comparing them with those without PA in the sputum. Finally, the low number of females in our cohort of COPD patients is concordant with previous studies in our area and probably related with the low prevalence of tobacco use among females for many years [11, 29].

Our study shows that the majority of PA isolates remain susceptible to most antipseudomonal agents, including fluoroquinolones. We would concur with recent guidelines that suggest these drugs be considered as empirical treatment for patients with the worst functional impairment [12, 21], but variations in the antibiotic resistance profiles in different geographic areas should be contemplated. The present study was not designed to evaluate the utility of specific antimicrobial treatment in persistence of PA and, therefore, we cannot specify whether their use can improve the prognosis in these patients. We have commented previously that a high percentage of the PA disappears without antibiotic treatment [26], and the higher number of re-admissions in the PA group seems to be more related with a greater severity of the disease than with failure of the antibiotic treatment. Future research leading to better understanding of the role of the specific antimicrobial treatment in these patients is needed.

In summary, we have shown the high prevalence of PA isolation in sputum in patients hospitalised for AECOPD. PA is associated with the worst functional parameters, systemic corticosteroid treatment, previous hospital admissions, worse values of BODE index and previous isolation of PA.

\section{SUPPORT STATEMENT}

This study was supported by a research grant from Fundació per la reçerca de Mútua de Terrassa (Barcelona, Spain) (MI03/07).

\section{STATEMENT OF INTEREST}

None declared.

\section{REFERENCES}

1 Cabello H, Torres A, Celis R, et al. Bacterial colonisation of distal airways in healthy subjects and chronic lung disease: a bronchoscopic study. Eur Respir J 1997; 10: 1137-1144.
2 Zalacain R, Sobradillo V, Amilibia J, et al. Predisposing factors to bacterial colonization in chronic obstructive pulmonary disease. Eur Respir J 1999; 13: 343-348.

3 Rosell A, Monso E, Soler N, et al. Microbiologic determinants of exacerbation in chronic obstructive pulmonary disease. Arch Intern Med 2005; 165: 891-897.

4 Sethi S, Evans N, Grant B, et al. New strains of bacteria and exacerbations of chronic obstructive pulmonary disease. $N$ Engl J Med 2002; 347: 465-471.

5 Papi A, Bellettato CM, Braccioni F, et al. Infections and airway inflammation in chronic obstructive pulmonary disease severe exacerbations. Am J Respir Crit Care Med 2006; 173: 1114-1121.

6 Wilkinson TM, Patel IS, Wilks M, et al. Airway bacterial load and FEV1 decline in patients with chronic obstructive pulmonary disease. Am J Respir Crit Care Med 2003; 167: 1090-1095.

7 Hurst JR, Perera WR, Wilkinson TM, et al. Systemic and upper and lower airway inflammation at exacerbation of chronic obstructive pulmonary disease. Am J Respir Crit Care Med 2006; 173: 71-78.

8 Miravitlles M, Espinosa C, Férnandez-Laso E, et al. Relationship between bacterial flora in sputum and functional impairment in patients with acute exacerbations of COPD. Chest 1999; 116: 40-46.

9 Lode H, Allewelt M, Balk S, et al. A prediction model for bacterial etiology in acute exacerbations of COPD. Infection 2007; 35: 143-149.

10 Alamoudi OS. Bacterial infection and risk factors in outpatients with acute exacerbation of chronic obstructive pulmonary disease: a 2-year prospective study. Respirology 2007; 12: 283-287.

11 Almagro P, Barreiro B, Ochoa de Echagüen A, et al. Risk factors for hospital readmission in patients with chronic obstructive pulmonary disease. Respiration 2006; 73: 311-317.

12 Woodhead M, Blasi F, Ewing S, et al. Guidelines for the management of adult lower respiratory tract infections. Eur Respir J 2005; 26: 1138-1180.

13 Eller J, Ede A, Schaberg T, et al. Infective exacerbations of chronic bronchitis: relation between bacteriologic etiology and lung function. Chest 1998; 113: 1542-1548.

14 Groenewegen $\mathrm{KH}$, Wouters EFM. Bacterial infections in patients requiring admission for an acute exacerbation of COPD; a 1-year prospective study. Respir Med 2003; 97: 770-777.

15 Ko F, Ip M, Chan P, et al. A 1-year prospective study of the infectious etiology in patients hospitalized with acute exacerbations of COPD. Chest 2007; 131: 44-152.

16 Anthonisen NR, Manfreda J, Warren CP, et al. Antibiotic therapy in exacerbations of chronic obstructive pulmonary disease. Ann Intern Med 1987; 106: 196-204.

17 Standardization of spirometry: 1994 update. American Thoracic Society. Am J Respir Crit Care Med 1995; 152: 1107-1136.

18 ATS Committee on Proficiency Standards for Clinical Pulmonary Function Laboratories, ATS statement: guidelines for the sixminute walk test. Am J Respir Crit Care Med 2002; 166: 111-117.

19 Celli BR, Cote CG, Marin JM, et al. The body-mass index, airflow obstruction, dyspnea, and exercise capacity index in chronic obstructive pulmonary disease. N Engl J Med 2004; 350: 1005-1012.

20 Lin SH, Kuo PH, Hsueh PR, et al. Sputum bacteriology in hospitalized patients with acute exacerbation of chronic obstructive pulmonary disease in Taiwan with an emphasis on Klebsiella pneumoniae and Pseudomonas aeruginosa. Respirology 2007; 12: 81-87.

21 Pauwels RA, Buist AS, Calverley P, et al. Global strategy for the diagnosis, management and prevention of chronic obstructive disease. NHLBI/WHO global initiative for chronic obstructive disease. Am J Respir Crit Care Med 2001; 163: 1256-1276.

22 Ong KC, Earnest A, Lu SJ. A multidimensional grading system (BODE index) as predictor of hospitalization for COPD. Chest 2005; 128: 3810-3816.

23 Imfeld S, Bloch KE, Weder W, et al. The BODE index after lung volume reduction surgery correlates with survival. Chest 2006; 129: 873-878. 
24 Patel IS, Vlahos I, Wilkinson TM, et al. Bronchiectasis, exacerbation indices, and inflammation in chronic obstructive pulmonary diseases. Am J Respir Crit Care Med 2004; 170: 400-407.

25 O'Brien C, guest PJ, Hill SL, et al. Physiological and radiological characterisation of patients diagnosed with chronic obstructive pulmonary disease in primary care. Thorax 2000; 55: 635-642.

26 Murphy TF, Brauer AL, Eschberger K, et al. Pseudomonas aeruginosa in chronic obstructive pulmonary disease. Am J Respir Crit Care Med 2008; 177: 853-860.
27 Monso E, Ruiz J, Rosell A, et al. Bacterial infection in chronic obstructive pulmonary disease. A study of stable and exacerbated outpatients using the protected specimen brush. Am J Resp Crit Care Med 1995; 152: 1316-1320.

28 Soler N, Agustí C, Angrill J, et al. Bronchoscopic validation of the significance of sputum purulence in severe exacerbations of chronic obstructive pulmonary disease. Thorax 2007; 62: 29-35.

29 Garcia-Aymerich J, Farrero E, Félez MA, et al. Risk factors of readmission to hospital for a COPD exacerbation: a prospective study. Thorax 2003; 58: 100-105. 